\title{
Tamizaje fitoquímico preliminar y actividad Antimicrobiana in vitro del extracto etanolico de la corteza de Hippocratea excelsa Kunth (Cancerina)
}

\author{
Preliminary phytochemical screening and in vitro antimicrobial activity of the bark of \\ Hippocratea excelsa Kunth (Cancerin)
}

\author{
Georgina Almaguer-Vargas ${ }^{a}$, Daniela F. Pacheco-Miramontes ${ }^{b}$, Perutsi M. Rivera-Cabrera ${ }^{c}$ \\ Marco A. Becerril-Flores ${ }^{d}$ José L. Imbert-Palafox ${ }^{e}$, Eva M. Molina-Trinidad ${ }^{f}$, Carmen \\ Balderas-Delgadillo $^{g}$, Mirna E. Ruiz-Anaya ${ }^{h}$, José R. Montejano-Rodríguez $^{i}$
}

\begin{abstract}
:
The Hippocratea excelsa Kunth (cancerine) plant is widely used for its antimicrobial properties in infectious processes in traditional Mexican medicine; however, there are few scientific papers to support it. Therefore, the objective of this work was to evaluate the antibacterial effect of the ethanol extract of Hippocratea excelsa Kunth to provide evidence about this effect. Methods: The antimicrobial-disk diffusion test, (Kirby Bauer), and a preliminary qualitative phytochemical-phytochemical test were performed. Results: The ethanol extract of cancerine at a dose of 3000mg managed to moderately inhibit the growth of Staphylococcus aureus, Streptococcus pyogenes and Staphylococcus epidermidis, with inhibition halos of $4.5 \mathrm{~mm}, 2.6 \mathrm{~mm}$ and $2.8 \mathrm{~mm}$ respectively. In the phytochemical test were found in the ethanol extract: alkaloids, quinones, coumarins, saponins, resins, phenols, tannins, flavonoids and bitter principles. Discussion: The antibacterial activity of some plants has been attributed to the presence of various compounds, including alkaloids, simple phenols, tannins, and flavones; Hippocratea excelsa Kunth presents this type of metabolites, however, the antimicrobial effect was not very significant, compared with the control (commercial) antibiogram. The metabolites found in the ethanol extract of the bark of Hippocratea excelsa Kunth matches with the phytochemical studies of the compounds present in the genus, reported by other research.
\end{abstract}

\section{Keywords:}

Hippocratea excelsa Kunth, phytochemistry, Kirby-Bauer, Staphylococcus, Streptococcus.

\section{Resumen:}

La planta Hippocratea excelsa Kunth (cancerina) es usada ampliamente por sus propiedades antimicrobianas en procesos infecciosos en la medicina tradicional mexicana, sin embargo, existen pocos trabajos científicos que lo avalen. Por lo tanto, el objetivo del presente trabajo fue evaluar el efecto antibacteriano del extracto etanólico de Hippocratea excelsa Kunth para aportar evidencia acerca de dicho efecto. Métodos: El ensayo antimicrobiano- prueba de difusión en disco, (Kirby Bauer), y un ensayo

\footnotetext{
a Instituto de Ciencias de la Salud, Área Académica de Farmacia, Universidad Autónoma del Estado de Hidalgo, Pachuca de Soto, Hidalgo., México. Email: almaguergina@gmail.com

${ }^{\mathrm{b}}$ Centro de Estudios Científicos y Tecnológicos Hidalgo. (CECyT 16) Instituto Politécnico Nacional, Pachuca de Soto, Hidalgo., México. Email: danielapacheco_m@hotmail.com

Centro de Estudios Científicos y Tecnológicos Hidalgo. (CECyT 16) Instituto Politécnico Nacional, Pachuca de Soto, Hidalgo., México. Email: perutsigeymer@gmail.com

d Instituto de Ciencias de la Salud, Área Académica de Medicina, Universidad Autónoma del Estado de Hidalgo, Pachuca de Soto, Hidalgo., México. Email: mbecerril_65@yahoo.com

Instituto de Ciencias de la Salud, Área Académica de Medicina, Universidad Autónoma del Estado de Hidalgo, Pachuca de Soto, Hidalgo., México. Email. imbertox@ hotmail.com

${ }^{\mathrm{f}}$ Instituto de Ciencias de la Salud, Área Académica de Medicina, Universidad Autónoma del Estado de Hidalgo, Pachuca de Soto, Hidalgo., México. Email: emolinatrinidad@gmail.com

${ }^{g}$ Instituto de Ciencias de la Salud, Área Académica de Odontología, Universidad Autónoma del Estado de Hidalgo, Pachuca de Soto, Hidalgo., México. Email: carbaldel@gmail.com

${ }^{\mathrm{h}}$ Instituto de Ciencias de la Salud, Área Académica de Farmacia, Universidad Autónoma del Estado de Hidalgo, Pachuca de Soto, Hidalgo., México. Email: mirna_ruiz10517@uaeh.edu.mx

Autor de correspondencia. Instituto de Ciencias de la Salud, Área Académica de Farmacia, Universidad Autónoma del Estado de Hidalgo, Pachuca de Soto, Hidalgo., México. Email: jrmontejano@yahoo.com.mx
} 
fitoquímico - fitoquímico cualitativo preliminar se realizaron. Resultados: El extracto etanólico de cancerina a dosis de 3000mg logro inhibir moderadamente el crecimiento de Staphylococcus aureus, Streptococcus pyogenes y Staphylococcus epidermidis, con halos de inhibición de $4.5 \mathrm{~mm}, 2.6 \mathrm{~mm}$ y $2.8 \mathrm{~mm}$ respectivamente. En el ensayo fitoquímico se encontraron en el extracto etanolico: alcaloides, quinonas, cumarinas, saponinas, resinas, fenoles, taninos, flavonoides y principios amargos. Discusión: La actividad antibacteriana de algunas plantas ha sido atribuida a la presencia de diversos compuestos, entre los que se incluyen alcaloides, fenoles simples, taninos, y flavonas; Hippocratea excelsa Kunth presenta este tipo de metabolitos, sin embargo, el efecto antimicrobiano no fue muy significativo, comparándolo con el antibiograma control (comercial). Los metabolitos encontrados en el extracto etanolico de la corteza de Hippocratea excelsa Kunth coinciden con los estudios fitoquímicos de los compuestos presentes en el género, reportados por otras investigaciones.

\section{Palabras Clave:}

Hippocratea excelsa Kunth, Fitoquímica, Kirby-Bauer, Staphylococcus, Streptococcus.

\section{Introducción}

La resistencia bacteriana a los antibióticos se considera una de las mayores amenazas para la salud mundial, algunas de sus consecuencias son: mayor tiempo en estancias hospitalarias, incremento de costos médicos y de la mortalidad, ${ }^{1}$ motivo por el cual la OMS considera que este problema se ha venido consolidando como uno de los temas en salud más preocupantes de los últimos años. ${ }^{2}$

Esta resistencia producto de la mutación bacteriana en respuesta al uso de antibióticos ha superado la velocidad de desarrollo y producción de nuevas moléculas con efecto antimicrobiano, por lo cual frecuentemente surgen procesos infecciosos para los cuales no existe un tratamiento eficaz. $^{2}$

Por lo anteriormente dicho es necesario continuar con la búsqueda de nuevas sustancias antimicrobianas que ofrezcan alternativas terapéuticas.

La OMS plantea como una opción para garantizar la atención sanitaria promover la utilización segura y eficaz de la Medicina Tradicional. Esta ha constituido la base para el desarrollo de la medicina moderna y representa una de las principales fuentes de atención sanitaria, es accesible, asequible, además es culturalmente aceptada y a veces es la única en muchas zonas rurales e indígenas y se constituye como el único recurso disponible. ${ }^{3}$ La República Mexicana es uno de los cinco países con mayor biodiversidad. Actualmente se han registrado cerca de 4,000 especies con atributos medicinales. ${ }^{4,5}$ Por lo tanto es importante el estudio de los metabolitos presentes en las plantas medicinales, así como su efecto antimicrobiano.

En México, la planta Hippocratea excelsa Kunth (Cancerina) de la familia de las Celastraceae, género Hippocratea, se utiliza en la medicina tradicional contra diversas infecciones en heridas, ulceras cutáneas y ulceras vaginales; disentería y entre otros usos contra cáncer, así como ectoparasiticida en pediculosis. ${ }^{6,7}$ En estudios realizados con el extracto acuoso y etanolico de Hippocratea excelsa, Alanis y colaboradores (2005) reportan una fuerte actividad antimicrobiana al probarla contra Escherichia coli; Shigella sonnei; Shigella flexneri; y Salmonella. ${ }^{8}$

Dado lo anteriormente mencionado el objetivo de este trabajo fue identificar la actividad antibacteriana in vitro así como realizar la identificación fitoquímica cualitativa del extracto etanolico de la corteza de $H$. excelsa.

\section{Metodología}

Las pruebas fitoquímicas permiten conocer los compuestos químicos presentes en los materiales vegetales a estudiar y son punto clave para la detección de compuestos con posible actividad antimicrobiana. Así mismo, las pruebas de difusión en disco permiten determinar la susceptibilidad bacteriana a determinada sustancia y se complementan para brindar información referente a la actividad antimicrobiana del compuesto a probar.

El experimento, se desarrollo en dos fases: la primera, relacionada con la obtención del extracto etanolico de la corteza y el tamizaje fitoquímico; la segunda, con la aplicación de las pruebas microbiológicas.

\section{Material vegetal y obtención de los extractos:}

La corteza de $\mathrm{H}$. excelsa se compró por internet con las características: orgánica y deshidratada; de la marca tierra de colores, s/ lote, teniendo como vendedor al Sr. Juan Sánchez; dicha corteza se fragmento con un molino de mano en fracciones de aproximadamente $2 \mathrm{~cm}$, por cada $100 \mathrm{~g}$ de corteza se agregó un litro de etanol al $70 \%$. Se vertió en dos frascos ámbar los cuales se sellaron herméticamente y se protegieron de la luz solar directa forrándolos con papel aluminio.

Se agitaron manualmente dos veces al día durante 7 días a temperatura ambiente. ${ }^{9}$

Transcurridas dos semanas, el macerado se filtró. La solución se sometió a destilación al vacío con rotavapor a temperatura de $40^{\circ} \mathrm{C}$ y una presión de 170 mbar para su extracción, obteniéndose el extracto crudo de la 
corteza.

\section{Fitoquímica cualitativa del extracto etanolico}

El tamizaje fitoquímico preliminar de la corteza de $\mathrm{H}$. excelsa se realizó con pruebas colorimétricas o de precipitación cualitativas después de evaporar los extractos. Las pruebas utilizadas para la determinación fueron alcaloides (Dragendorff, Mayer, Wagner), triterpenos y esteroides (Liebermann-Burchard, Solkowski, Rosemheim), cumarinas (Baljet, Legal, Erlich), saponinas (espuma) Resinas, aceites esenciales y sustancias grasas (papel blanco, sudan IV), fenoles y taninos (cloruro férrico), Flavonoides (ácido sulfúrico concentrado, Shinoda), principios amargos y astringentes (sabor). Los resultados fueron interpretados como negativos 0 positivos $(+$ poco; ++moderado; +++ abundante). ${ }^{10,11}$

\section{Pruebas microbiológicas}

Se trabajó con cepas bacterianas de referencia de Pseudomona aeruginosa ATCC 10145, Klebsiella oxytoca ATCC 8729, Staphylococcus aureus ATCC 25923, Enterococcus faecalis ATCC 29212, Escherichia coli ATCC 25922, Proteus vulgaris ATCC 49132, Streptococcus pyogenes ATCC 19615, Candida albicans ATCC 10231 y Streptococcus pneumoniae ATCC 49136, las cuales se resembraron en caldo nutritivo (tripticasa soya) y se incubaron en estufa 24 horas a $37^{\circ} \mathrm{C}$. De las bacterias cultivadas en el caldo nutritivo se tomaron 0.5 $\mathrm{ml}$ y se diluyeron en solución salina, hasta igualar al patrón de turbidez del tubo 0,5 de la escala de Mc Farland.

Posteriormente, se efectuó una siembra masiva sobre agar Mueller Hinton con la ayuda de un hisopo, el cual se pasó de manera uniforme sobre la superficie del agar; se colocaron sensidiscos impregnados con el extracto obtenido, a concentraciones de 20, 50 100, 1000, 2000 y $3000 \mu \mathrm{g} / 10 \mu \mathrm{L}$ y grupos control con sensidiscos comerciales de la marca Multibac I.D (Tabla 1), se procedió a la incubar, a $37^{\circ} \mathrm{C}$ por 24 horas, para observar la posible presencia de halos de inhibición (Las pruebas se realizaron por triplicado). ${ }^{12}$

\begin{tabular}{|c|c|c|}
\hline Clave & Antibiótico & Concentración \\
\hline AK & Amikacina & $30 \mathrm{mcg}$ \\
\hline \multirow[t]{5}{*}{ AM } & Ampicilina & $10 \mathrm{mcg}$ \\
\hline & Enterobacteriaceae & \\
\hline & Staphylococcus sp. & \\
\hline & Enterococos & \\
\hline & Estreptococos & \\
\hline \multirow[t]{3}{*}{ CB } & Carbenicilina & $100 \mathrm{mcg}$ \\
\hline & Enterobacteriaceae & \\
\hline & Pseudomonas sp & \\
\hline CF & Cefalotina & $30 \mathrm{mcg}$ \\
\hline CFX & Cefotaxima & $30 \mathrm{mcg}$ \\
\hline CTZ & Ceftazidima & $30 \mathrm{mcg}$ \\
\hline CTX & Ceftriaxona & $30 \mathrm{mcg}$ \\
\hline CXM & Cefuroxima & $30 \mathrm{mcg}$ \\
\hline CPF & Ciprofloxacina & $5 \mathrm{mcg}$ \\
\hline CLM & Clindamicina & $30 \mathrm{mcg}$ \\
\hline CL & Cloranfenicol & $30 \mathrm{mcg}$ \\
\hline \multirow[t]{2}{*}{ DC } & Dicloxacilina & $1 \mathrm{mcg}$ \\
\hline & Staphylococcus sp. & \\
\hline ENX & Enoxacina & $10 \mathrm{mcg}$ \\
\hline $\mathbf{E}$ & Eritromicina & $15 \mathrm{mcg}$ \\
\hline GE & Gentamicina & $10 \mathrm{mcg}$ \\
\hline NET & Netilmicina & $30 \mathrm{mcg}$ \\
\hline NF & Nitrofurantoína & $300 \mathrm{mcg}$ \\
\hline NOF & Norfloxacina & $10 \mathrm{mcg}$ \\
\hline \multirow[t]{6}{*}{ PE } & Penicilina & $10 U$ \\
\hline & Enterococos & \\
\hline & Estreptococos & \\
\hline & Staphylococcus sp. & \\
\hline & N. gonorrhoeae & \\
\hline & Neumococos & \\
\hline SXT & $\begin{array}{l}\text { Sulfametoxazol } \\
\text { Trimetoprim }\end{array}$ & $25 \mathrm{mcg}$ \\
\hline
\end{tabular}




\begin{tabular}{cll}
\hline TE & Tetraciclina & $\mathbf{3 0 ~ m c g}$ \\
\hline VA & Vancomicina & $30 \mathrm{mcg}$ \\
& Enterococos \\
& Staphylococcus \\
& aureus & \\
\hline $\begin{array}{l}\text { Tabla 1.- } \\
\text { comerciales. }\end{array}$
\end{tabular}

\section{Resultados}

Tras la elaboración del extracto etanolico de $\mathrm{H}$. excelsa se encontró que el rendimiento fue del $5.981 \%$.

Fitoquímica cualitativa

Las pruebas realizadas al extracto etanolico revelaron conforme a los parámetros establecidos, una mayor cantidad de alcaloides y saponinas; presencia moderada de triterpenos, esteroides, cumarinas, resinas y flavonoides y escasa presencia de fenoles y taninos. (Tabla 2)

\begin{tabular}{|c|c|c|}
\hline METABOLITOS & ENSAYOS & $\begin{array}{c}\text { H. excelsa } \\
\text { E. Etanolico }\end{array}$ \\
\hline \multirow{3}{*}{ Alcaloides } & Dragendorff & +++ \\
\hline & Mayer & +++ \\
\hline & Wagner & +++ \\
\hline \multirow{3}{*}{$\begin{array}{l}\text { Triterpenos y } \\
\text { Esteroides }\end{array}$} & Solkowski & ++ \\
\hline & $\begin{array}{l}\text { Lieberman- } \\
\text { Burchard }\end{array}$ & - \\
\hline & Rosemheim & - \\
\hline \multirow{3}{*}{ Cumarinas } & Baljet & ++ \\
\hline & Legal & + \\
\hline & Erlich & - \\
\hline Saponinas & Espuma & +++ \\
\hline Resinas & Resinas & ++ \\
\hline $\begin{array}{l}\text { Fenoles y } \\
\text { Taninos }\end{array}$ & $\begin{array}{l}\text { Cloruro } \\
\text { Férrico }\end{array}$ & + \\
\hline \multirow{3}{*}{ Flavonoides } & $\begin{array}{l}\text { Ácido } \\
\text { Sulfúrico } \\
\text { concentrado }\end{array}$ & + \\
\hline & Shinoda & ++ \\
\hline & Rosemheim & ++ \\
\hline $\begin{array}{l}\text { Principios } \\
\text { amargos y } \\
\text { astringentes }\end{array}$ & Sabor & +++ \\
\hline
\end{tabular}

Tabla 2.- Fitoquímica preliminar de $H$. excelsa (+ bajo; ++ medio; +++ alto)

\section{Pruebas de sensibilidad bacteriana}

La prueba de difusión en disco mostro que el extracto etanolico de $\mathrm{H}$. excelsa presenta actividad antimicrobiana contra Streptococcus pneumoniae ATCC 49136, Streptococcus. pyogenes ATCC 19615 y Staphylococcus aureus ATCC 25923.

Tabla 3. Sensibilidad antimicrobiana del extracto etanolico de $H$. excelsa

\begin{tabular}{|l|c|}
\hline \multicolumn{1}{|c|}{ Agente patógeno } & $\begin{array}{l}\text { Diámetro del Halo } \\
\text { de inhibición (mm) }\end{array}$ \\
\hline $\begin{array}{l}\text { Pseudomona aeruginosa ATCC } \\
10145\end{array}$ & 0.0 \\
\hline $\begin{array}{l}\text { Streptococcus pneumoniae } \\
\text { ATCC 49136 }\end{array}$ & 2.8 \\
\hline $\begin{array}{l}\text { Staphylococcus aureus ATCC } \\
25923\end{array}$ & 2.6 \\
\hline Klebsiella oxytoca ATCC 8729 & 0.0 \\
\hline $\begin{array}{l}\text { Enterococcus faecalis ATCC } \\
29212\end{array}$ & 0.0 \\
\hline Escherichia coli ATCC 25922 & 0.0 \\
\hline Proteus vulgaris ATCC 49132 & 0.0 \\
\hline Candida albicans ATCC 10231 & 0.0 \\
\hline $\begin{array}{l}\text { Streptococcus pyogenes ATCC } \\
19615\end{array}$ & 4.5 \\
\hline
\end{tabular}

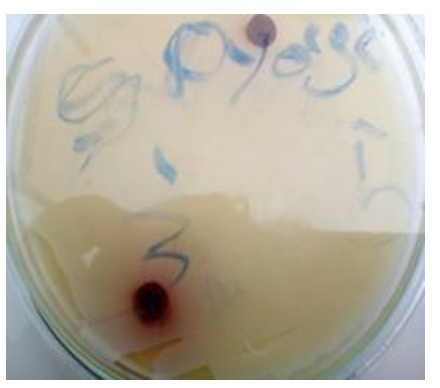

Figura 6. S.aureus

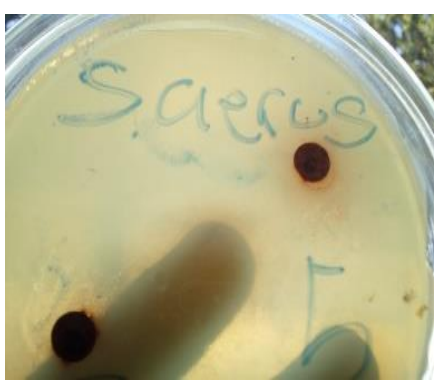

Figura 7. S.pyogenes

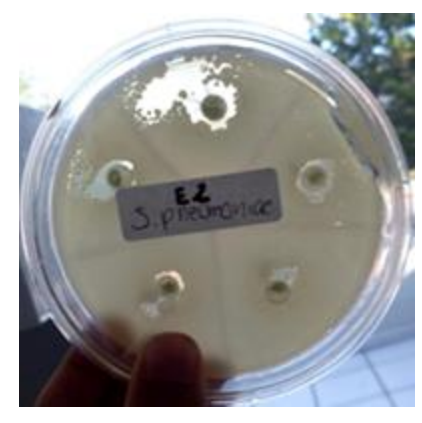

Figura 8. S. pneumoniae 


\section{Discusión}

La resistencia bacteriana es un grave problema de salud pública por lo que es necesario buscar nuevas fuentes de obtención de antibióticos para ofrecer alternativas terapéuticas antibacterianas. Los antibióticos han estado presentes desde hace millones de años, antes que los humanos empezaran a poblar el planeta y las fuentes de obtención de los mismos son muy variadas. Bajo este enfoque, las plantas ofrecen una excelente alternativa para la búsqueda de este tipo de compuestos, no obstante, se requieren más estudios en estas para conocer su potencial antimicrobiano.

$H$. excelsa se utiliza de forma tradicional para tatar infecciones por la población mexicana, específicamente cicatrización de heridas en piel y ulceras vaginales. ${ }^{6} \mathrm{Su}$ uso es tan extendido que se comercializa en mercados en México y por internet. ${ }^{13}$

De los trabajos en los cuales se ha probado la actividad antimicrobiana de los extractos metanolicos y acusosos de Hippocratea excelsa, Alanis y colaboradores ${ }^{8}$ estudiaron dicho efecto contra enterobacterias causantes de diarrea y disentería en la población mexicana, trabajando con dos cepas de Salmonella spp., dos cepas de E. coli., dos cepas de Shigella sonnei y Shigella flexneri; demostrando actividad antimicrobiana, la cual fue mayor en los extractos metanolicos que en los acuosos, resultando superior a la ejercida por cloranfenicol, pero menor a la mostrada por Trimetoprim.

Las pruebas de difusión en disco llevadas a cabo por nuestro equipo de trabajo fueron negativas contrastadas con los controles de cloranfenicol y trimetoprim, al determinar la sensibilidad antimicrobiana de Salmonella spp., Shigella y E Coli, a diferentes concentraciones (20, $50100,1000,2000$ y $3000 \mu \mathrm{g} / 10 \mu \mathrm{L}$ ) con el extracto etanolico de la misma planta. Por lo tanto, los resultados obtenidos en el presente trabajo no coinciden con los obtenidos por Alanís y colaboradores. ${ }^{8}$ Cabe destacar que el extracto y la concentración utilizada por Alanís y colaboradores fueron diferentes a las usadas en el presente trabajo, lo cual puede ser factor para la discrepancia entre los resultados obtenidos sobre bacterias Gram negativas. Así mismo, los metabolitos activos presentes en el extracto etanolico probado en el presente trabajo parecen mostrar una mayor actividad sobre bacterias Gram positivas.

Por otra parte, Fuente Mata probó extractos etanolicos de Hippocratea excelsa, Glychirriza glabra y Urtica dioica en bacterias de interés odontológico, utilizando el mismo método de difusión en disco, reportando actividad antimicrobiana contra Streptococcus mutans y Streptococcus sobrinus, pero solo de Glychirriza glabra y Urtica dioica a concentraciones de 500 y $1000 \mu \mathrm{g} / \mathrm{ml}$, sin embargo, no encontró resultados con el extracto de Hippocratea excelsa. ${ }^{14}$

Las pruebas de sensibilidad in vitro, llevadas a cabo por nuestro equipo de trabajo para determinar la susceptibilidad de Streptococcus pyogenes y Streptococcus pneumoniae, así como Staphylococcus aureus a diferentes concentraciones (20, 50 100, 1000, 2000 y $3000 \mu \mathrm{g} / 10 \mu \mathrm{L}$ ) del extracto etanolico de Hippocratea excelsa mostraron cierta sensibilidad a la concentración de $3000 \mu \mathrm{g} / 10 \mu \mathrm{L}$, actividad manifiesta por la presencia de halos de inhibición de $4.5 \mathrm{~mm}, 2.6$ $\mathrm{mm}$ y $2.8 \mathrm{~mm}$ respectivamente. Nuestros resultados pueden hacer referencia a un posible efecto bacteriostático del extracto sobre el crecimiento tanto de Streptococcus como de Staphylococcus, contrario a los resultados obtenidos para el género Streptococcus, por Fuente Mata. La marcada presencia de alcaloides contenidos en el extracto etanolico de Hippocratea excelsa, pudiera sugerir que son este tipo de compuestos los que estén involucrados en el efecto antimicrobiano bacteriostático ya que se trata de compuestos altamente distribuidos en el reino vegetal y a los cuales se les atribuye actividad antimicrobiana de bajo y amplio espectro. ${ }^{21}$ Poseen una actividad biológica y una diversidad estructural muy variada, y una de las clasificaciones más destacadas, es la de los alcaloides isoquinolínicos y bis- bencilisooquinolínicos, que han sido reportados como los metabolitos secundarios de mayor actividad antibacteriana, en plantas superiores. ${ }^{22}$ De la presencia y abundancia de este y otro tipo de alcaloides depende la actividad antimicrobiana mostrada por el extracto a probar y puede ser que la extracción con etanol no arrastre este tipo de compuestos en las cantidades adecuadas para mostrar un efecto antimicrobiano mas marcado, o que nuestra muestra vegetal no contenga dichos compuestos en abundancia, y que por lo tanto nuestros resultados solo muestren actividad parcial contra bacterias Gram positivas y un efecto nulo contra las especies Gram negativas probadas en este estudio (Pseudomona aeruginosa., Klebsiella oxytoca., Enterococcus faecalis y Proteus vulgaris) con respecto a los controles Ciprofloxacino, Vancomicina, Penicilina, Ampicilina, Trimetoprim y Tetraciclina.

La presencia de triterpenos, ${ }^{15},{ }^{17}$ alcaloides, ${ }^{16,} 20$ flavonoides, $^{18}$ quinonas $^{20}$, cumarinas, saponinas, resinas, principios amargos y astringentes, fenoles y taninos, ${ }^{19}$ se corresponden con los reportados para el género por otros trabajos de investigación, y de estos, los que cuentan con actividad antimicrobiana reportada, son: alcaloides, saponinas quinonas, taninos, cumarinas y

flavonoides. 
Es necesario llevar a cabo de manera concomitante a la prueba de difusión en disco, otras pruebas que complementen los resultados obtenidos en el presente trabajo, tales como micro dilución en pozo, dilución en agar, Epsilon test (E test), macro dilución en caldo, entre otras; así mismo consideramos necesario llevar a cabo las pruebas necesarias para determinar la concentración minina inhibitoria, del extracto.

Se recomienda continuar con el trabajo experimental con miras a aislar y caracterizar los diferentes metabolitos presentes en esta especie, para poder llevar a cabo ensayos mejor dirigidos que pongan de manifiesto la actividad biológica específica de dichos compuestos, esto con la finalidad de determinar si estas sustancias pueden ser usados como antibióticos o si pueden ser candidatos para modificación química en laboratorio para crear nuevas sustancias con actividad antimicrobiana.

\section{Conclusiones}

La actividad antibacteriana preliminar mostrada por el extracto etanolico de Hippocratea excelsa va de acuerdo con los usos etnobotánicos que se le han dado.

Los metabolitos encontrados en el extracto etanolico de la corteza de $H$. excelsa se corresponden con los reportados para el género y fueron: alcaloides, quinonas, cumarinas, saponinas, resinas, fenoles, taninos, flavonoides, principios amargos y astringentes. De estos, los que cuentan con actividad antimicrobiana reportada, son: alcaloides, saponinas quinonas, taninos, cumarinas y flavonoides.

El efecto antimicrobiano de Hippocratea excelsa a las concentraciones de 20, 50 100, 1000, $2000 \mu \mathrm{g}$ contra Pseudomona aeruginosa, Klebsiella oxytoca, Enterococcus faecalis, Escherichia coli, Proteus vulgaris y Candida albicans fue negativo.

La concentración de $3000 \mu \mathrm{g}$ mostro una ligera respuesta antimicrobiana contra Streptococcus pyogenes, Staphylococcus aureus y Streptococcus pneumoniae representada por halos de inhibición de $4.5 \mathrm{~mm}, 2.6 \mathrm{~mm}$ y $2.8 \mathrm{~mm}$ respectivamente.

\section{Referencias}

[1] OMS. Organización Mundial de la Salud. Centro de prensa. Resistencia a los antibióticos. 5 de febrero de 2018.

[2] Ponce de León-Rosales S, Arredondo-Hernández R y López-Vidal Y. La resistencia a los antibióticos: Un grave problema global. Gac Med Mex. 2015; 151: 681-9.
[3] OMS. Organización Mundial de la Salud. Estrategias de la OMS sobre Medicina Tradicional 2014-2023.2013; 1:16-17. http://apps.who.int/ medicinedocs/es/d/Js2299s/

[4] Jiménez Sierra CL, Sosa Ramírez J, Cortés-Calva P, Solís Cámara AB, Íñiguez Dávalos LI y Ortega-Rubio A. México país mega diverso y la relevancia de las áreas naturales protegidas. 2014; 60: 16-22.

[5] Estrada Muñiz E, Guerrero-Palomo G y Vega L. Natural Products: News anti-cáncer agents derived from plants. 2012; 8: 19-20.

[6] Maldonado Almanza BJ, Alemán Octaviano AM, Gadea Noguerón R. y Rangel AltamiranoMG. Plantas útiles de la Mixteca Baja Poblana. Morelos: Universidad Autónoma del Estado de Morelos. 2017. $1^{\mathrm{a}}$ ed.

[7] Reyes-Chilpa R, Jiménez-Estrada M, Cristóbal-Telésforo E, TorresColín L, Villavicencio MA, Pérez-Escandón BE y Mercado-González R. Natural Insecticides from Hippocratea excelsa and Hippocratea celastroides. Economic Botany. 2003; 57(1):54-64. Retrieved from http://www.jstor.org/stable/4256642

[8] Alanís AD, Calzada F, Cervantes JA, Torres J, Ceballos GM. Antibacterial properties of some plants used in Mexican traditional medicine for the treatment of gastrointestinal disorders. J Ethnopharmacol. 2005; 100(1-2):153-7.

[9] Arroyo J, Almora Y, Quino M, Raez E, Martínez J, Buendía J, Baca D, Hañari R. Efecto protector en cirrosis hepática inducida en ratas del extracto etanólico de las hojas de Piper aduncum comparado con silimarina. Anales de la Facultad de Medicina. 2012; 73 (2):85-91.

[10] Ochoa Pacheco A, Escalona Arranz JC, Fechine Tavares J, Sobral Da Silva M. Evaluación fitoquímica de hojas de Excoecaria lucida Sw. (Aité) (Euphorbiaceae) y Aislamiento e Identificación de un hemiterpenoide. Revista Cubana de Plantas Medicinales. 2015; 20(1):117-130. Recuperado en 01 de julio de 2019, de http://scielo.sld.cu/scielo.php?script=sci_arttext\&pid=S102847962015000100011\&lng=es\&tlng=es.

[11] Martínez N, Almaguer G, Vázquez-Alvarado P, Figueroa A, Zúñiga C, Hernández-Ceruelos A. Análisis fitoquímico de Jatropha dioica y determinación de su efecto antioxidante y quimioprotector sobre el potencial genotóxico de ciclofosfamida, daunorrubicina y metilmetanosulfonato evaluado mediante el ensayo cometa. Boletín Latinoamericano y del Caribe de Plantas Medicinales y Aromáticas. 2014; 13 (5): 437-457.

[12] Hudzicki J. Kirby-bauer disk diffusion susceptibility test protocol. http://www.asmscience.org/content/education/protocol/proto col.3189?crawler=redirect. 2009. Consultado 12 Junio 2018.

[13] Mendoza CG. Cancerina. Hippocreatea excelsa H.B.K. en Plantas medicinales en los mercados de México. México Universidad Autónoma de Chapingo. 2011; $1^{\circ}$ Ed. 226-227.

[14] Fuente Mata J. Evaluación de extractos de Hippocratea excelsa, Glychirriza glabra y Urtica dioica en bacterias de interés odontológico. Maestría tesis, Universidad Autónoma de Nuevo León. 2018. Repositorio Académico Digital. UANL. Obtenido de http://eprints.uanl.mx/16303/

[15] Cáceres-Castillo D, Mena-Rejón G.J, Cedillo-Rivera R., Quijano L.D. 21ß-Hydroxy-oleanane-type triterpenes from Hippocratea excelsa. 2008.

[16] Furukawa M, Makino M, Uchiyama T, Ishimi K, Ichinohe Y, Fujimoto Y. Sesquiterpene pyridine alkaloids from Hippocratea $\begin{array}{llll}\text { excelsa. } & \text { Phytochemistry. } 2002 \quad \text { Apr; } & \text { 59(7):767-77 }\end{array}$ 
Publicación semestral, Educación y Salud Boletín Científico Instituto de Ciencias de la Salud Universidad Autónoma del Estado de

Hidalgo, Vol. 8, No. 14 (2019) 41-47

[17] Mena-Rejón GJ, Pérez-Espadas AR, Moo-Puc RE, Cedillo-Rivera R, Bazzocchi IL, Jiménez-Díaz IA, Quijano L. Antigiardial activity of triterpenoids from root bark of Hippocratea excelsa.J Nat Prod. 2007; 70(5):863-5.

[18] Navarrete A, Trejo-Miranda JL, Reyes-Trejo L. Principles of root bark of Hippocratea excelsa (Hippocrataceae) with gastroprotective activity. J Ethnopharmacol. 2002; 79(3):383-8.

[19] Villa -Fernández MGJ, Barajas-Morales J. Estudio Anatómico De Hippocratea Excelsa Hbk. (Hippocrateaceae) Acta Botánica Mexicana. 1998; 43:7-21.

[20] Déciga-Campos M, Rivero-Cruz I, Arriaga-Alba M, CastáñedaCorral G, Angeles-López GE, Navarrete A, Matab R. Acute toxicity and mutagenic activity of Mexican plants used in traditional medicine. Journal of Ethnopharmacology. 2007; 110 334-342.

[21] Patiño Ladino, OJ, Prieto Rodríguez, JA, Lozano Moreno, JM, Lesmes Sarmiento, L, Cuca Suárez, LE. Propiedades antibacterianas in vitro de metabolitos secundarios aislados de dos especies del género Zanthoxylum (Rutaceae). Rev Cubana Farm. 2011; 45 (3).

[22] Peña López, DH. Evaluación de la actividad antibacteriana de los alcaloides provenientes de las hojas de siparuna sessiliflora. Tesis. Pontificia Universidad Javeriana. Facultad De Química. 2011. 